Original Article

\title{
Effects of vibratory stimulation-induced kinesthetic illusions on the neural activities of patients with stroke
}

Takayuki Kodama, PhD, $\mathrm{PT}^{1)^{*}}$, Hideki Nakano, PhD, PT ${ }^{2)}$, Hironori Ohsugi, PhD, $\mathrm{PT}^{1)}$, Shin Murata, PhD, $\mathrm{PT}^{1)}$

1) Department of Physical Therapy, Faculty of Health Sciences, Kyoto Tachibana University:

34 Yamada-cho, Oyake, Yamashina-ku, Kyoto 607-8175, Japan

2) Department of Neurorehabilitation, Graduate School of Health Sciences, Kio University, Japan

\begin{abstract}
Purpose] This study evaluated the influence of vibratory stimulation-induced kinesthetic illusion on brain function after stroke. [Subjects] Twelve healthy individuals and 13 stroke patients without motor or sensory loss participated. [Methods] Electroencephalograms were taken at rest and during vibratory stimulation. As a neurophysiological index of brain function, we measured the $\mu$-rhythm, which is present mainly in the kinesthetic cortex and is attenuated by movement or motor imagery and compared the data using source localization analyses in the Standardized Low Resolution Brain Electromagnetic Tomography (sLORETA) program. [Results] At rest, $\mu$-rhythms appeared in the sensorimotor and supplementary motor cortices in both healthy controls and stroke patients. Under vibratory stimulation, no $\mu$-rhythm appeared in the sensorimotor cortex of either group. Moreover, in the supplementary motor area, which stores the motor imagery required for kinesthetic illusions, the $\mu$-rhythms of patients were significantly stronger than those of the controls, although the $\mu$-rhythms of both groups were reduced. Thus, differences in neural activity in the supplementary motor area were apparent between the subject groups. [Conclusion] Kinesthetic illusions do occur in patients with motor deficits due to stroke. The neural basis of the supplementary motor area in stroke patients may be functionally different from that found in healthy controls. Key words: Kinesthetic illusion, Stroke, Supplementary motor area
\end{abstract}

(This article was submitted Sep. 14, 2015, and was accepted Oct. 30, 2015)

\section{INTRODUCTION}

In the field of neurorehabilitation, kinesthetic illusion ${ }^{1)}$ has been used as a rehabilitation therapy for patients who have suffered a stroke. Kinesthetic illusion is used to create the impression of limb movement in the brain, without patients actually having to perform the movement voluntarily ${ }^{1}$. Kinesthetic illusions, which are induced by sensorimotor information in the brain, involve the excitement of sensorimotor cortical areas and the association of underlying neuronal activity with motion control and skill learning is believed to have beneficial effects in rehabilitation ${ }^{2}$.

The therapeutic effects of kinesthetic illusion on the motor deficits of patients with stroke have been reported in a number of publications ${ }^{3,4)}$. Most studies have employed mirrors to induce kinesthetic illusion ${ }^{5,6)}$. For example, Altschuler et al. ${ }^{7)}$ demonstrated that mirror-induced kinesthetic illusions resulted in recovery of motor function even in patients in the chronic phase of post-stroke recovery (i.e., 6 months or longer after the occurrence of stroke). In addition, Dohle et al. ${ }^{8)}$ examined the effects of a mirror-based treatment on patients suffering severe paralysis after stroke and found improvements in finger motor function after the intervention.

When an intervention task entails processing visual information during kinesthetic illusion, various types of brain func-

*Corresponding author. Takayuki Kodama (E-mail: kodama-t@tachibana-u.ac.jp)

(C2016 The Society of Physical Therapy Science. Published by IPEC Inc.

This is an open-access article distributed under the terms of the Creative Commons Attribution Non-Commercial No Derivatives (by-nc-nd) License $<$ http://creativecommons.org/licenses/by-nc-nd/4.0/>. 
tions, such as cognitive, visual information processing, and motor functions, related to the unaffected extremity can affect performance of the task. Therefore, the stimulus used in the task itself should be as simple and passive as possible. Thus, some studies have used vibratory stimulation $3,9,10)$ as a method for inducing kinesthetic illusion.

Functional magnetic resonance imaging ${ }^{10)}$ and magnetoencephalography ${ }^{11}$ ) have implicated the following regions in kinesthetic illusion-related neural activity: the primary sensorimotor cortex, the sensory association area, the supplementary motor area, and the angular gyrus. During kinesthetic illusion, subjects predict a sensation even before motion occurs. Given that previous sensorimotor experiences need to be simulated in kinesthetic motor imagery ${ }^{12}$, it is important that brain function be analyzed based on the degree of kinesthetic illusion achieved. Therefore, in order to determine the brain functions occurring during such an illusion, detailed temporal and spatial analyses (from the beginning of kinesthetic motor image formation) need to be performed before the induced kinesthetic illusion occurs.

The electroencephalographic mu-rhythm ${ }^{13}$ ( $\mu$-wave) is useful as a neurophysiological index for capturing data from such analyses. A $\mu$-wave is the high-frequency component ranging from 10 to $13 \mathrm{~Hz}$ of the alpha band ${ }^{14)}$, which appears in the sensorimotor cortex ${ }^{13,15)}$ and the supplementary motor area ${ }^{16,17)}$. Since this wave is attenuated not only by actual motion, but also by kinesthetic motor imagery ${ }^{13,15)}$, it reflects neural activity both during kinesthetic illusion and during the generation of a motor image. To date, the association between kinesthetic illusion and $\mu$-waves has been reported for healthy subjects ${ }^{18)}$, but not for stroke patients.

Thus, the purpose of this study was to examine the impact of kinesthetic illusion, produced by vibratory stimulation, on the cerebral neural activity of patients with stroke, through functional neuroimaging of $\mu$-waves.

\section{SUBJECTS AND METHODS}

\section{Subjects}

In accordance with the Declaration of Helsinki, the scope and contents of this study, as well as the precautions against the disclosure of personal information, were explained to the subjects verbally and in written form. Upon their understanding of the explanations, subjects provided their written informed consent by signing the relevant documents. The study was approved by the Ethics Committee of Kurume University School of Medicine.

This study was conducted on 12 healthy subjects (six men and six women; mean age: $60.1 \pm 3.2$ years) with no orthopedic or nervous system disease, or motor deficits or sensory disorders (the healthy group), and 13 patients with stroke (seven men and six women; mean age: $61.1 \pm 3.8$ year; the patient group). The dominant hands of the subjects in the healthy group were assessed using the Edinburgh Inventory ${ }^{19}$, and all subjects were confirmed to be right-handed. We confirmed that none of the subjects in the patient group had any cognitive dysfunction or disorder, or any sensory impairment. The attributes of the

Table 1. Attributes of the patient group

\begin{tabular}{lc}
\hline Characteristics of patients & \\
\hline Age, yrs (range) & $61.1 \pm 3.8(56-68)$ \\
Male:Female (n) & $7: 6(\mathrm{n}=13)$ \\
Diagnosis & \\
$\quad$ cerebral infarction & \\
$\quad$ lacunar & 2 \\
$\quad$ thalamus & 2 \\
$\quad$ internal capsule & 4 \\
$\quad$ corona radiata & 1 \\
$\quad$ atherothrombosis & \\
$\quad$ cerebral hemorrhage & 2 \\
$\quad$ thalamus & 2 \\
$\quad$ putamen & \\
Paralysis & 13 \\
right & 0 \\
left & \\
Dominant hand & \\
right & 13 \\
left & 0 \\
Time from injury, months & $12.6 \pm 7.6$ \\
UE-FMA & $21.8 \pm 5.6$ \\
MMSE & $26.9 \pm 1.8$ \\
\hline UE-FMA: Fugl-Meyer Assesment (UE), MMSE: Mini-Mental State Exami- \\
nation
\end{tabular}


patients are shown in Table 1.

\section{Electroencephalography}

The subjects were instructed to relax while reclining on a chair that had a backrest with an adjustable angle, and were asked to position the forearm of their left upper extremity (that is, the upper extremity on the non-measured side) so that the elbow touched the chair. It was first explained to subjects that electroencephalographic measurements would be performed under two conditions: a resting state, the control condition (rest), and during the application of a vibratory stimulation, the task condition (vibratory stimulation).

The motion targeted in the vibratory stimulation-induced kinesthetic illusion was extension of the right elbow joint. Therefore, the bicep tendon of the right upper arm was selected as the stimulation site. Because the stimulation was applied to the skin, cutaneous stimulus receptors, in addition to muscle spindles, were excited. The $\mu$-waves, which change according to sensorimotor information, are also influenced by sensory information received from cutaneous stimuli ${ }^{20)}$; therefore, as a control, the subjects were asked to envision that elbow flexion was prevented due to contraction of the bicep muscle during vibratory stimulation ${ }^{20)}$. In order to ensure that the forearm would not be in contact with the trunk, the measurer fixed a hand massager (THRIVE MD-01; Thrive Co., Ltd., Osaka, Japan) on the bicep tendon of the subjects' right arms.

At rest, electroencephalographic measurements were conducted without applying any vibratory stimulation. During the stimulation, vibrations with a frequency of $91.7 \mathrm{~Hz}^{1,10,21)}$ were applied. In addition, since visual information pertaining to the non-moving upper extremity has been found to reduce kinesthetic illusion, subjects were instructed to close their eyes during the vibratory stimulation ${ }^{9)}$.

Data were collected for 3 to 9 days (mean $4.9 \pm 2.0$ days). Vibratory stimulation and rest assessment were both carried out twice a day for 3 consecutive or non-consecutive days. After-effects were taken into consideration by allowing subjects to rest between vibratory stimulation sessions. Thus, the measurements were obtained in a total of twelve sessions. The average value of the brain wave data of the twelve measurements was calculated, and used as the data for each subject.

Electroencephalographic measurements were performed during the two conditions, and a Neurofax EEG-1200 (Nihon Kohden, Tokyo, Japan) was used to record the electroencephalograms. Measurements were conducted at 18 sites of the international 10-20 system (F3, F4, F7, F8, Fz, C3, C4, Cz, P3, P4, Pz, O1, O2, Oz, T3, T4, T5, and T6), with the earlobes on both sides as the reference electrodes. Data were processed with a bandpass filter of 0.5 to $60 \mathrm{~Hz}$, and the sampling frequency was $1,000 \mathrm{~Hz}$. The duration of the measurement was set to $60 \mathrm{~s}$ per session for both the rest and vibratory stimulation conditions ${ }^{10)}$.

\section{Data analysis}

For the imaging analysis, 30 epochs were extracted from the data obtained under each condition (the duration of one epoch was $2 \mathrm{~s}$ ), and a frequency analysis was performed. The data within the $\mu$-wave frequency range $(10 \mathrm{Ho} 13 \mathrm{~Hz})$ were calculated and used for intracerebral spatial analyses using the Standardized Low Resolution Brain Electromagnetic Tomography (sLORETA) analysis ${ }^{22}$, a method that allows three-dimensional image display of intracerebral neural activity. The values of each voxel in the brain areas displaying neural activity under each condition were calculated as the neural activity (current source density [CSD]; $\mu \mathrm{A} / \mathrm{mm}^{2 *} 10^{-3}$ ), and were identified in terms of Brodmann's area (BA) and Montreal Neurological Institute (MNI) coordinates ${ }^{23}$. By averaging data using the sLORETA Averager software, the neural activity could be calculated as the global field power (GFP) value ${ }^{22)}$ in the two groups under both the rest and vibratory stimulation conditions. In addition, the intracerebral neural activities of the two groups under each condition were compared using the statistics in sLORETA $\mathrm{SnPM}^{24}$ ). In this analysis, the neural areas showing significant differences in activity (with a statistical significance level of 5\%) were colored, calculated, and drawn. The significance threshold was based on a permutation test with 5000 permutations, using log-transformed LORETA values, with subject-wise normalization (on the assumption that differences in the base-line activity between healthy subjects and patients may exist). When $\mu$-waves manifest in the sensorimotor cortex ${ }^{13)}$, frontal lobe ${ }^{25}$, or occipital lobe ${ }^{26}$, the waveform components and the names of the waves can differ (such as $\alpha$-waves) depending on their region of occurrence. Interestingly, this phenomenon is evident even when these waves have the same frequency range. Thus, in this study, the different types of $\mu$-waves were investigated separately.

\section{RESULTS}

Prior to the analysis, participants were asked what type of feeling they sensed in the elbow joint upon vibratory stimulation, and 23 of the 25 initially recruited subjects answered: "I felt as though my elbow kept extending continuously," and "I felt as though the elbow continued to move toward the opposite side by itself." This confirmed that kinesthetic illusion was successfully induced. However, for two participants in the patient group, a kinesthetic illusion could not be confirmed. Therefore, the data of the 12 subjects in the healthy group and the remaining 11 subjects in the patient group were used for the analysis.

The MNI coordinates and CSD values of the areas showing significant differences between the groups under each condition. In the healthy group, intracerebral neural activity in the $\mu$-wave frequency range was observed at rest in an extended region of motor area BA4 and sensory areas BA3, 2, and 1 in the sensorimotor cortex, as well as in supplementary motor 
area BA6. On the other hand, $\alpha$-waves were observed in an extended region of the prefrontal cortex and occipital lobe, which showed significantly higher neural activity than other areas of the cerebral cortex (Fig. 1-a). In the patient group, significantly stronger $\mu$-wave activity was found in the sensorimotor cortical areas, and the supplementary motor area BA6, while $\alpha$ waves were observed in an extended region of the occipital lobe (Fig. 1-b). In comparison to the patient group, healthy individuals showed a significant increase in neural activity in sensorimotor cortical areas, supplementary motor area BA6, the occipital lobe, and the prefrontal area (Fig. 1-c).

In both groups, intracerebral neural activity of the $\mu$-wave frequency band during vibratory stimulation showed significantly higher activity in the occipital lobe than in other areas of the cerebral cortex; however, sensorimotor cortical areas showed no significant activity in the $\mu$-wave frequency band during neural activity (Fig. 2-a, b). In comparison to the patient group, the healthy group showed a significant increase in activity in the occipital lobe, while the patient group exhibited greater activity in the supplementary motor area BA6 (Fig. 2-c).

\section{DISCUSSION}

This study examined the impact of kinesthetic illusions induced by vibratory stimulation on the intracerebral neural activity in patients with stroke, using functional neuroimaging analysis of electroencephalographic $\mu$-waves as a neurophysiological index.

At rest, the patient group showed $\mu$ - and $\alpha$-waves in the sensorimotor cortical areas, prefrontal areas, and in an extended area over the occipital lobe. These results were consistent with the conventionally known origins of $\mu$-waves, namely, the sensorimotor cortical areas ${ }^{13)}$, frontal lobe ${ }^{25)}$, and occipital lobe ${ }^{26)}$. The presence of $\mu$-waves at rest reflected the inhibition of neuronal excitation in sensorimotor areas, and may also reflect the resting state similar to $\alpha$-waves ${ }^{25)}$. Therefore, our results confirm previous reports of brain waves in the frequency range of 10 to $13 \mathrm{~Hz}$ at rest. Our findings also indicate the reliability of the electroencephalographic parameters measured in this study.

A comparison of the control and patient groups at rest showed that $\alpha$-waves were significantly decreased in the prefrontal cortex and in the occipital lobe in the patient group compared to the healthy group. In general, the production of $\alpha$-waves decreases with age ${ }^{27)}$ or stroke ${ }^{28)}$. Recently, Dubovik, et al. ${ }^{29)}$ reported that ischemic lesions caused by stroke decrease the

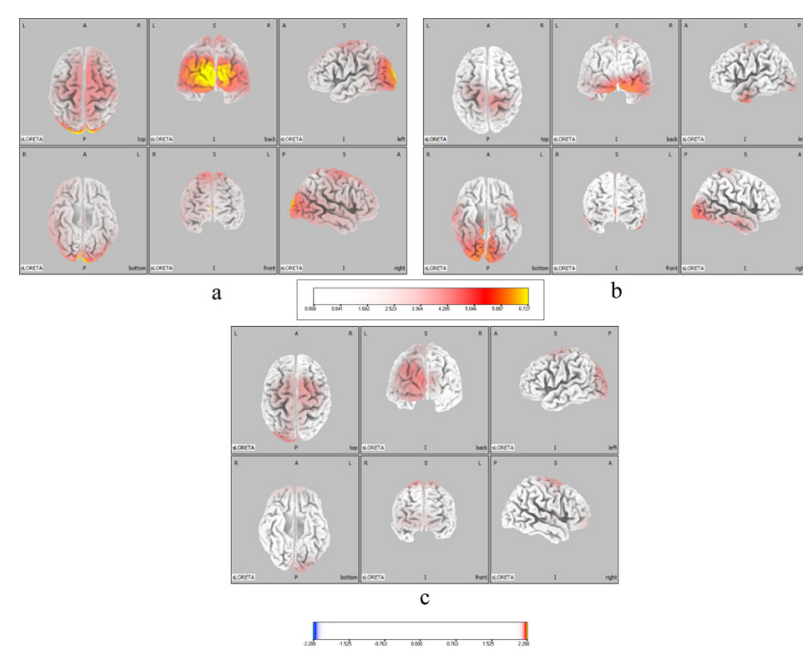

Fig.1. Neural activity in the $\mu$-wave range at rest

a: Healthy group

b: Patient group

Areas of the brain where the $\mu$ - and $\alpha$-waves significantly increased are indicated in yellow and red.

c: Comparison of the neural activities of the healthy and patient groups

Areas of the brain where the $\mu$ - and $\alpha$-waves significantly increased compared to the patient group are indicated in yellow and red. Color scale values below the images indicate $t$-values $(t=2.288)$

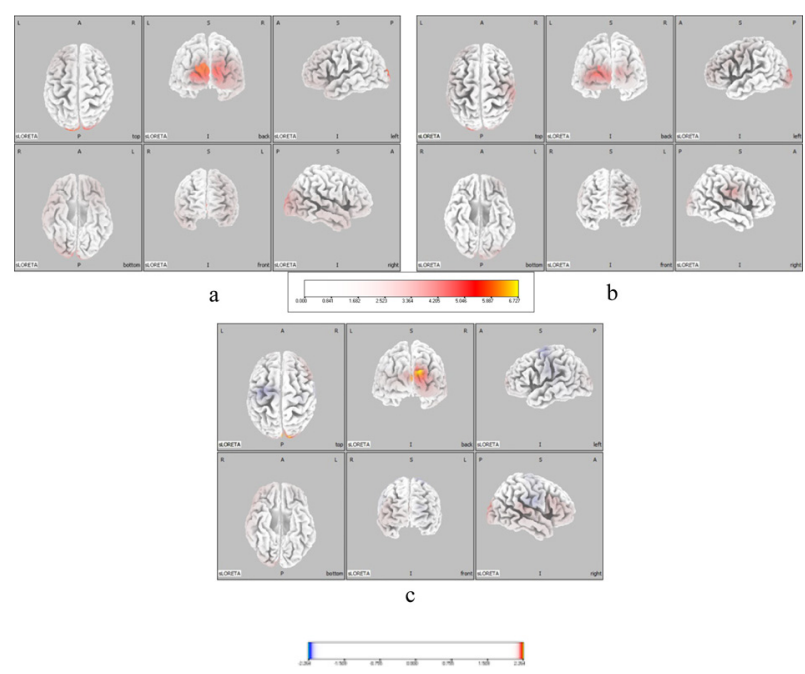

Fig. 2. Neural activity in the $\mu$-wave range during vibratory stimulation

a: Neural activity in the $\mu$-wave range of the healthy group b: Neural activity in the $\mu$-wave range of the patient group Brain areas where the $\mu$ - and $\alpha$-waves significantly increased are indicated in yellow and red.

c: Comparison of the neural activities of the healthy and patient groups

Areas of brain where the $\mu$ - and $\alpha$-waves significantly increased in the healthy group compared to the patient group are indicated in yellow and red, and areas of the brain where the $\mu$ - and $\alpha$-waves significantly increased in the patient group compared to the healthy group are indicated in light blue and blue. Color scale values below the images indicate $t$-values $(t=2.264)$ 
synchrony of $\alpha$-waves. Moreover, they concluded that this decrease is related to the cognitive and motor deficits observed in stroke patients. In addition, as a characteristic property of electroencephalograms in patients with stroke, the laterality of $\alpha$-waves is not limited to the damaged site, but is often found in the sensorimotor cortical areas and occipital lobe. In the thalamus, $\alpha$-waves are suppressed when the anteromedial region is damaged ${ }^{30)}$. The lateral edges of lesions in many of our participants were located in the anterior and mediolateral regions of the thalamus. This may have contributed to the significant reductions in $\mu$ - and $\alpha$-waves seen in the patient group compared to the healthy group.

Furthermore, in the sensorimotor cortex and supplementary motor area BA6, $\mu$-waves were significantly decreased in the patient group compared to the healthy group. BA6 conducts oriented processing of information ${ }^{31,32)}$ for the sensorimotor cortical areas, suggesting that BA6 may functionally be closely related to the sensorimotor cortex. The neural activity in these areas is known to involve a decrease in $\mu$-waves during preparation for motor execution, the so-called "idling" rhythm ${ }^{33)}$. Therefore, the stroke-induced decrease of $\alpha$-waves might include $\mu$-waves. However, our findings suggest that motor impairment may have affected the process from the stage before the integration of sensorimotor information aimed at executing motion, and that the neural basis of those regions may be functionally different from those of the healthy group.

During vibratory stimulation, intracerebral neural activity in the $\mu$-wave frequency band did not change significantly in the sensorimotor areas of either subject group. This suggests an increase in information from the sensorimotor cortical areas, that plays an important role in the processing of kinesthetic information from the muscle spindles, and in motor perception ${ }^{3)}$. In this case, $\mu$-waves may have been attenuated because of the asynchronous nature of neuronal activity on the two sides ${ }^{34)}$ in the sensorimotor cortical areas ${ }^{13,15)}$. Neural activity in the motor area BA4 is not only involved in the processing of sensory information from the muscle spindle, but is also related to sensorimotor information per $\mathrm{se}^{3}$. Such information includes the kinesthetic illusion that results from the process; thus, kinesthetic illusions are greater when the excitation of the motor area is more extensive ${ }^{2}$. Our results therefore indicate that kinesthetic illusions triggered by vibratory stimuli increased neural activity in the sensorimotor cortical areas.

A comparison of the two groups found that activity in BA6 significantly increased during vibratory stimulation in the patient group compared to the healthy group. Previous studies have shown that, in patients with stroke, $\mu$-waves are decreased compared to healthy subjects ${ }^{35}$. In addition, our study revealed differences in the neural activity of the supplementary motor area BA6, but not in the motor area BA4, which is responsible for perception during kinesthetic illusions. Therefore, there may be differences between the healthy subjects and patients in terms of processing of neural information during the generation of kinesthetic illusions. BA6 is involved in spatial cognition of sensorimotor information ${ }^{36}$ ), not only during actual movement, but also during kinesthetic illusion. This area stores simulations of motor imagery based on previous sensorimotor experiences, which are needed for kinesthetic illusion ${ }^{12}$, and it is considered to be the area that discriminates sensorimotor information ${ }^{37,38)}$. Therefore, it could be hypothesized that patients with stroke functionally differ from healthy individuals in terms of the generation of kinesthetic illusions, and particularly at the simulation stage or in the storage of motor imagery.

In this study, electroencephalographic analysis was found to be a useful tool for reflecting ${ }^{38)}$ brain functioning after stroke. We examined the effects of kinesthetic illusion on intracerebral neural activity in patients after stroke using functional neuroimaging analysis based on $\mu$-waves. Our findings suggest that kinesthetic illusions occur in patients with motor deficits due to stroke, and that such patients exhibit elevated neural activity in the sensorimotor areas and in supplementary motor area BA6. Our study shows the importance of motor imagery in kinesthetic illusion. However, this study did not compare or verify neural activity in the sensorimotor, supplementary motor, or related areas. Moreover, motor imagery ability or motor function of the subjects was no evaluated. In future, the functional association between sensorimotor areas and related brain regions will require further detailed examination using electroencephalographic analysis. More specifically, these analyses will need to investigate the induction and generation of kinesthetic illusion and neural activity in sensorimotor cortical areas. It will also be important to quantitatively compare healthy subjects and patients with stroke with respect to their abilities to recall motor images, and to assess the association of this process with activity in the supplementary motor area.

\section{REFERENCES}

1) Goodwin GM, McCloskey DI, Matthews PB: The contribution of muscle afferents to kinaesthesia shown by vibration induced illusions of movement and by the effects of paralysing joint afferents. Brain, 1972, 95: 705-748. [Medline] [CrossRef]

2) Naito E, Roland PE, Ehrsson HH: I feel my hand moving: a new role of the primary motor cortex in somatic perception of limb movement. Neuron, 2002, 36: 979-988. [Medline] [CrossRef]

3) Imai R, Hayashida K, Nakano H, et al.: Brain activity associated with the illusion of motion evoked by different vibration stimulation devices: An fNIRS Study. J Phys Ther Sci, 2014, 26: 1115-1119. [Medline] [CrossRef]

4) Schaechter JD, Perdue KL: Enhanced cortical activation in the contralesional hemisphere of chronic stroke patients in response to motor skill challenge. Cereb Cortex, 2008, 18: 638-647. [Medline] [CrossRef]

5) Ramachandran VS, Rogers-Ramachandran D, Cobb S: Touching the phantom limb. Nature, 1995, 377: 489-490. [Med- 
line] [CrossRef]

6) Park JY, Chang M, Kim KM, et al.: The effect of mirror therapy on upper-extremity function and activities of daily living in stroke patients. J Phys Ther Sci, 2015, 27: 1681-1683. [Medline] [CrossRef]

7) Altschuler EL, Wisdom SB, Stone L, et al.: Rehabilitation of hemiparesis after stroke with a mirror. Lancet, 1999, 353: 2035-2036. [Medline] [CrossRef]

8) Dohle C, Püllen J, Nakaten A, et al.: Mirror therapy promotes recovery from severe hemiparesis: a randomized controlled trial. Neurorehabil Neural Repair, 2009, 23: 209-217. [Medline] [CrossRef]

9) Hagura N, Takei T, Hirose S, et al.: Activity in the posterior parietal cortex mediates visual dominance over kinesthesia. J Neurosci, 2007, 27: 7047-7053. [Medline] [CrossRef]

10) Naito E, Ehrsson HH, Geyer S, et al.: Illusory arm movements activate cortical motor areas: a positron emission tomography study. J Neurosci, 1999, 19: 6134-6144. [Medline]

11) Casini L, Romaiguère $P$, Ducorps $A$, et al.: Cortical correlates of illusory hand movement perception in humans: a MEG study. Brain Res, 2006, 1121: 200-206. [Medline] [CrossRef]

12) Harada T, Saito DN, Kashikura K, et al.: Asymmetrical neural substrates of tactile discrimination in humans: a functional magnetic resonance imaging study. J Neurosci, 2004, 24: 7524-7530. [Medline] [CrossRef]

13) Bae SH, Jeong WS, Kim KY: Effects of mirror therapy on subacute stroke patients' brain waves and upper extremity functions. J Phys Ther Sci, 2012, 24: 1119-1122. [CrossRef]

14) Dornhege G, Benjamin B, Matthias K, et al.: Optimizing spatio-temporal filters for improving brain-computer interfacing. Adv Neural Inf Process Syst, 2005, 18: 315-322.

15) Kim SS, Lee BH: Measuring cerebral hemodynamic changes during action observation with functional transcranial doppler. J Phys Ther Sci, 2015, 27: 1379-1381. [Medline] [CrossRef]

16) Llanos C, Rodriguez M, Rodriguez-Sabate $C$, et al.: Mu-rhythm changes during the planning of motor and motor imagery actions. Neuropsychologia, 2013, 51: 1019-1026. [Medline] [CrossRef]

17) Aoki Y, Ishii R, Pascual-Marqui RD, et al.: Detection of EEG-resting state independent networks by eLORETA-ICA method. Front Hum Neurosci, 2015, 9: 31. [Medline] [CrossRef]

18) Evans N, Blanke O: Shared electrophysiology mechanisms of body ownership and motor imagery. Neuroimage, 2013, 64: 216-228. [Medline] [CrossRef]

19) Oldfield RC: The assessment and analysis of handedness: the Edinburgh inventory. Neuropsychologia, 1971, 9: 97-113. [Medline] [CrossRef]

20) Naito E, Nakashima T, Kito T, et al.: Human limb-specific and non-limb-specific brain representations during kinesthetic illusory movements of the upper and lower extremities. Eur J Neurosci, 2007, 25: 3476-3487. [Medline] [CrossRef]

21) Burke D, Hagbarth KE, Löfstedt L, et al.: The responses of human muscle spindle endings to vibration during isometric contraction. J Physiol, 1976, 261: 695-711. [Medline] [CrossRef]

22) Pascual-Marqui RD: Standardized low-resolution brain electromagnetic tomography (sLORETA): technical details. Methods Find Exp Clin Pharmacol, 2002, 24: 5-12. [Medline]

23) Collins DL, Holmes CJ, Peters TK, et al.: Automatic 3-D model-based neuroanatomical segmentation. Hum Brain Mapp, 1995, 3: 190-208. [CrossRef]

24) Pascual-Marqui RD: Instantaneous and lagged measurements of linear and nonlinear dependence between groups of multivariate time series: frequency decomposition. 2007, arXiv:0711.1455.

25) Pfurtscheller G: Event-related synchronization (ERS): an electrophysiological correlate of cortical areas at rest. Electroencephalogr Clin Neurophysiol, 1992, 83: 62-69. [Medline] [CrossRef]

26) Han D: Development of a brain index for dementia diagnosis using quantitative EEG analysis. J Phys Ther Sci, 2013, 25: 497-500. [CrossRef]

27) Lee B, Han D: Comparative analysis of brain activation between healthy elderly women and healthy adult women. $\mathrm{J}$ Phys Ther Sci, 2012, 23: 875-878. [CrossRef]

28) Dubovik S, Pignat JM, Ptak R, et al.: The behavioral significance of coherent resting-state oscillations after stroke. Neuroimage, 2012, 61: 249-257. [Medline] [CrossRef]

29) Dubovik S, Ptak R, Aboulafia T, et al.: EEG alpha band synchrony predicts cognitive and motor performance in patients with ischemic stroke. Behav Neurol, 2013, 26: 187-189. [Medline] [CrossRef] 
30) Andersen P, Eccles J: Inhibitory phasing of neuronal discharge. Nature, 1962, 196: 645-647. [Medline] [CrossRef]

31) Hong JH, Jang SH: Neural network related to hand movement: a combined study of diffusion tensor tractography and functional MRI. J Phys Ther Sci, 2011, 23: 97-101. [CrossRef]

32) Matsuda T, Watanabe S, Kuruma H, et al.: A comparison of three bimanual coordinations: an fMRI study. J Phys Ther Sci, 2009, 21: 85-92. [CrossRef]

33) Kuhlman WN: Functional topography of the human mu rhythm. Electroencephalogr Clin Neurophysiol, 1978, 44: 83-93. [Medline] [CrossRef]

34) Tanji J, Okano K, Sato KC: Neuronal activity in cortical motor areas related to ipsilateral, contralateral, and bilateral digit movements of the monkey. J Neurophysiol, 1988, 60: 325-343. [Medline]

35) Tecchio F, Zappasodi F, Pasqualetti P, et al.: Rhythmic brain activity at rest from rolandic areas in acute mono-hemispheric stroke: a magnetoencephalographic study. Neuroimage, 2005, 28: 72-83. [Medline] [CrossRef]

36) Boussaoud D: Attention versus intention in the primate premotor cortex. Neuroimage, 2001, 14: S40-S45. [Medline] [CrossRef]

37) Chung GH, Han YM, Jeong SH, et al.: Functional heterogeneity of the supplementary motor area. AJNR Am J Neuroradiol, 2005, 26: 1819-1823. [Medline]

38) Van de Winckel A, Sunaert S, Wenderoth N, et al.: Passive somatosensory discrimination tasks in healthy volunteers: differential networks involved in familiar versus unfamiliar shape and length discrimination. Neuroimage, 2005, 26: 441-453. [Medline] [CrossRef] 